\title{
DNA vaccines delivered by human papillomavirus pseudovirions as a promising approach for generating antigen-specific CD8+ T cell immunity
}

\author{
Shiwen Peng ${ }^{1}$, Barbara Ma', Shu-Hsia Chen ${ }^{5}$, Chien-Fu Hung ${ }^{1,3}$ and TC Wu ${ }^{1,2,3,4^{*}}$
}

\begin{abstract}
Background: Human papillomavirus (HPV) pseudovirions have recently been shown to deliver DNA efficiently in vivo, resulting in the priming of antigen-specific CD8+ T cells in vaccinated mice. In the current study, we compare the different preparation methods for the generation of HPV pseudovirions for their ability to efficiently infect cells. We also compare the antigen-specific CD8+ T cell immune responses generated by different DNA delivery methods and several commonly used forms of vaccination with that of HPV pseudovirions.

Results: We found that the preparation method of pseudovirions is important for the efficient delivery of encapsidated DNA. We have shown that vaccination with DNA encoding model antigen ovalbumin (OVA) delivered by HPV-16 pseudovirions was capable of generating therapeutic antitumor effects against OVA-expressing tumor. In addition, vaccination with DNA encoding OVA delivered by HPV-16 pseudovirions generated the highest number of OVA-specific CD8+ T cells in mice in our system compared to DNA delivered by other delivery methods. We also found that vaccination with OVA DNA delivered by HPV-16 pseudovirions generated the highest number of OVA-specific CD8+ T cells in mice compared to other forms of antigen-specific vaccines. Furthermore, HPV-16 pseudovirions were capable of carrying DNA vaccine encoding clinically relevant antigen, telomerase reverse transcriptase, to generate antigen-specific CD8+ T cell immune responses.

Conclusions: Our data suggest that DNA vaccines delivered by HPV-16 pseudovirions may be advantageous compared to other delivery methods and other forms of antigen-specific vaccines for application to antigenspecific immunotherapy.
\end{abstract}

\section{Background}

DNA vaccination has emerged as a promising way to generate antigen-specific $\mathrm{T}$ cell immunity due to its safety, stability, and capacity for repeated administration. However, naked DNA vaccines suffer from limited vaccine potency due to poor transfection efficiency in vivo. Therefore, an optimized and efficient delivery system that improves the transfection efficiency of DNA vaccines into cells in vivo may significantly improve the antigen-specific immunity generated by DNA vaccination for the control of virus-associated infections and/or tumors.

\footnotetext{
*Correspondence: wutc@jhmi.edu

'Department of Pathology, Johns Hopkins Medical Institutions, Baltimore, MD, USA

Full list of author information is available at the end of the article
}

We have recently introduced the use of replicationdefective human papillomavirus (HPV) pseudovirions as a novel approach to improve naked DNA vaccine delivery in vivo [1]. DNA plasmids can be packaged into the papillomavirus L1 and L2 capsid proteins to generate a 'pseudovirion' that can efficiently deliver the encapsidated DNA into infected cells. The encapsulation of the therapeutic DNA vaccine protects the DNA from nucleases and provides efficient targeted delivery with great stability. Additionally, because HPV pseudovirions contain a DNA construct with genes of interest, but not the natural HPV viral genome, they are non-replicative and lack many of the safety concerns associated with live viral vectors. Furthermore, neutralizing antibodies against one type of papillomavirus pseudovirion are usually not cross-reactive to other types of papillomavirus pseudovirions. The spectrum of over 100 different
C Biomed Central

() 2011 Peng et al; licensee BioMed Central Ltd. This is an Open Access article distributed under the terms of the Creative Commons Attribution License (http://creativecommons.org/licenses/by/2.0), which permits unrestricted use, distribution, and reproduction in any medium, provided the original work is properly cited. 
types of papillomavirus pseudovirions allows for repeated boosting with different types of HPV pseudovirions without concern for preexisting immunity. Thus, HPV pseudovirions represent a potentially safe gene delivery method for clinical usage.

We previously characterized human papillomavirus pseudovirions as an efficient delivery system for DNA vaccines in vivo [1]. We demonstrated that vaccination with HPV-16 pseudovirions containing a DNA vaccine encoding model antigen, ovalbumin (OVA), (HPV-16/ OVA psV) subcutaneously generated significantly stronger OVA-specific CD8+ T cell immune responses compared with OVA DNA vaccination via gene gun in a dose-dependent manner. We demonstrated that the L2 minor capsid protein was essential for the infectivity mediated by HPV-16/OVA psV. Additionally, we showed that papillomavirus pseudovirions are capable of infecting DCs [1]. Furthermore, the papillomavirus L1 capsid protein activates DCs to augment the immune response [2,3]. Thus, human papillomavirus pseudovirions represent an innovative and promising delivery system to trigger potent antigen-specific immune responses.

In the current study, we further characterize the application of HPV pseudovirions as an important method for the delivery of naked DNA immunization. We compared the method of preparing HPV pseudovirions for their ability to efficiently deliver DNA to cells. In addition, we analyzed the capability of HPV pseudovirions to deliver naked DNA to a bone marrow-derived dendritic cell line. Furthermore, we compared the delivery of DNA by HPV pseudovirions with other methods of administration and other forms of vaccines for their ability to generate antigen-specific $\mathrm{CD} 8+\mathrm{T}$ cell immune responses. Our data indicate that the method of preparing HPV pseudovirion is crucial for their ability to infect cells. In addition, DNA vaccines delivered by HPV pseudovirions are able to effectively be delivered to dendritic cells, resulting in potent antigen-specific CD8+ T cell immune responses compared to different delivery methods and other forms of vaccination. The potential clinical applications of HPV pseudovirion technology for delivery of naked DNA vaccine are discussed.

\section{Results \\ HPV pseudovirions prepared by intracellular assembly can infect cells with much greater efficiency than HPV pseudovirions prepared by in vitro assembly}

The preparation method for pseudovirions may be crucial to the efficiency of DNA delivery. It has been previously shown that naked DNA can be encapsidated by L1 and L2 capsid proteins using in vitro assembly $[4,5]$. The HPV structural proteins can spontaneously selfassemble into virus-like particles (VLPs) that morphologically resemble the native virions. These pseudovirions generated by in vitro assembly involve the disruption and refolding of HPV L1L2 VLPs $[4,5]$. In general, the preparation of pseudovirions by in vitro assembly is technically demanding and the pseudovirion generated via this mechanism lacks L2, which is required for infection. In comparison, a recent method involving intracellular assembly of papillomaviral vectors was shown to generate high titers of replication-defective papillomavirus pseudovirions for vaccination [6,7]. This intracellular assembly method provides packaging of the target plasmid within an L1 and L2 capsid. The production cell line, 293 cells, is engineered to express high levels of SV40 large T antigen (293TT) to drive the amplification of the target plasmid containing an SV40 origin of replication. These 293TT cells are co-transfected with codon-optimized L1 and L2 capsid genes in an expression vector that is too large for its efficient encapsidation along with the target plasmid (which can contain the SV40 origin of replication but it is not required), allowing for efficient intracellular production of HPV pseudovirions encapsidating DNA.

We therefore compared the infectivity of HPV-16 pseudovirions carrying GFP (HPV-16/GFP psV) prepared by in vitro assembly with the infectivity of HPV$16 /$ GFP psV prepared by intracellular assembly. As shown in Figure 1, the infectivity of HPV-16/GFP psV prepared by in vitro assembly was significantly lower compared to that of HPV-16/GFP psV prepared by intracellular assembly. These data suggest that this new method for the generation of large quantities of replication-defective HPV pseudovirions is a significantly more efficient method in comparison to the preparation of $\mathrm{HPV}$ pseudovirions by in vitro assembly.

\section{HPV-16 pseudovirions can efficiently infect dendritic cell line in vitro in a dose-dependent manner}

We have previously demonstrated that FITC-labeled HPV-16 pseudovirions carrying DNA encoding model antigen ovalbumin (HPV-16/OVA psV) were able to be taken up by $\mathrm{CD} 11 \mathrm{c}+$ dendritic cells in the draining lymph nodes of vaccinated mice [1]. To determine whether the dose of HPV pseudovirions correlates with the infectivity of DC-1 dendritic cell line, $5 \times 10^{4}$ of DC-1 cells/well were seeded into 24 -well plates the night before infection. The seeded DC-1 cells were then infected with increasing amounts of HPV-16/GFP psV (L1 protein ranging from 0 ug to $5 \mathrm{ug}$ ) for 72 hours and GFP expression was examined by flow cytometry. There was a clear correlation between percentage of GFP+ DC-1 cells and amount of L1 protein in HPV-16/GFP $\mathrm{psV}$, as demonstrated by the increase in GFP expression in $\mathrm{DC}-1$ cells with increasing amount of $\mathrm{L} 1$ protein in HPV-16/GFP psV (Figure 2A). As shown in Figure 2B, 


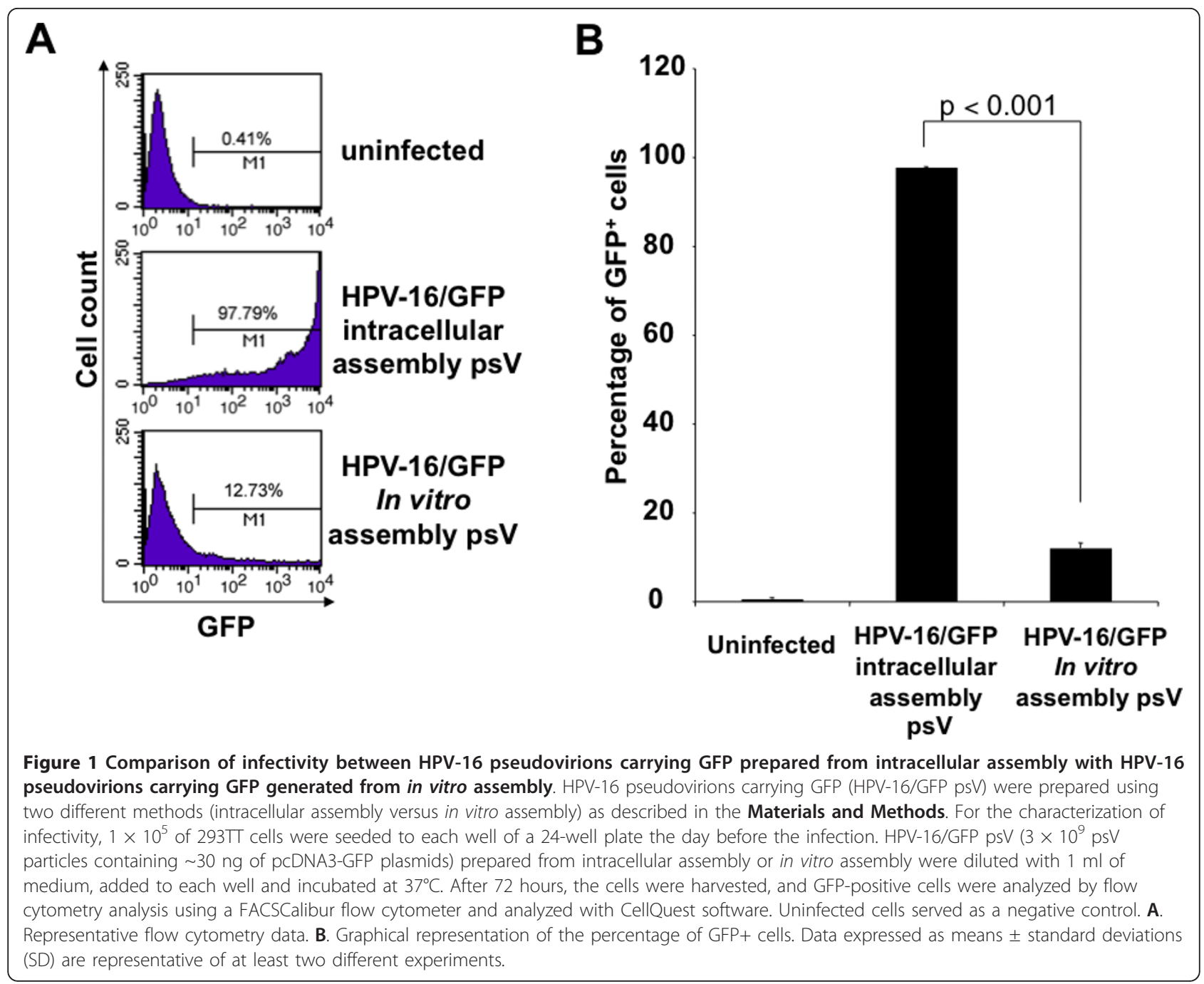

A

\section{B}
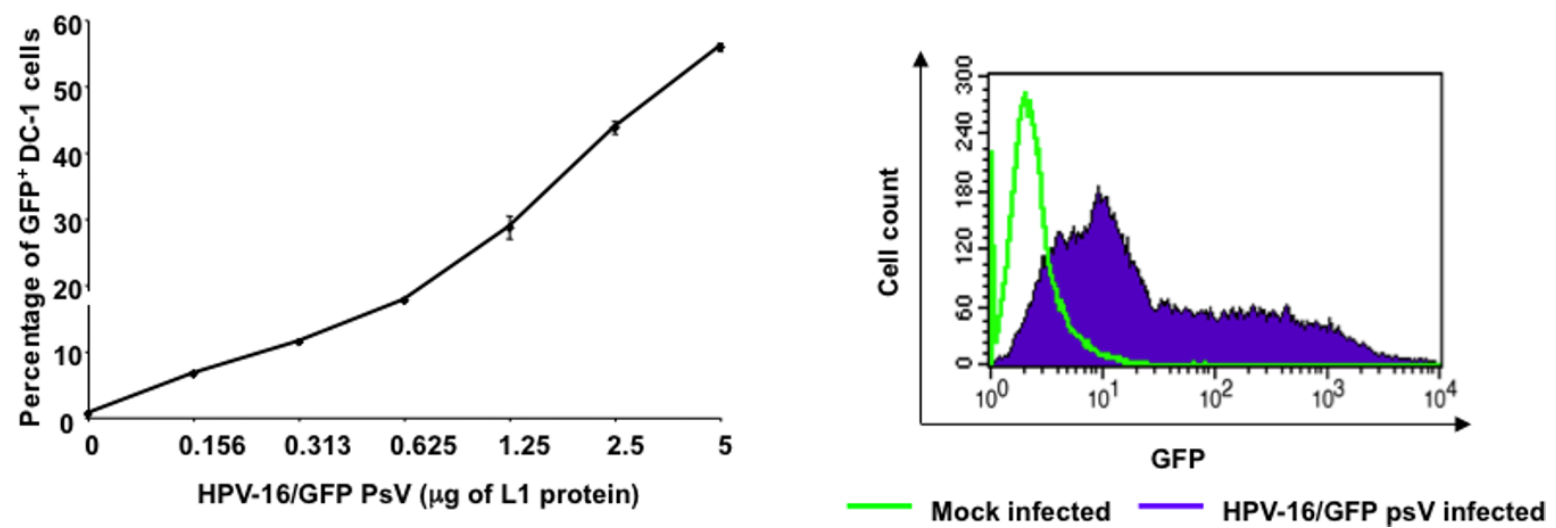

Figure 2 Infection of dendritic cells by HPV-16 pseudovirions. DC-1 cells $\left(5 \times 10^{4}\right.$ cells/well) were seeded into 24-well plate the night before infection. The seeded DC-1 cells were then infected with indicated amount of HPV16-GFP pseudoviruses (total amount of L1 protein) for 72 hours and GFP-positive cells were examined by flow cytometry. A. Line graph of percentage of GFP+ cells. B. Representative flow cytometry data of DC-1 cells infected with HPV-16/GFP psV (5 ug of L1 protein). 
DC-1 cells infected with HPV-16/GFP psV (5 ug L1 protein) showed a clear shift in the peak of GFP expression compared to that of uninfected cells, indicating that the majority of cells infected with HPV-16/GFP psVs had significantly greater GFP expression than uninfected cells. Thus, our data suggest that HPV pseudovirions can efficiently infect dendritic cells in vitro in a dose-dependent manner.

Treatment of tumor-bearing mice with DNA delivered by HPV-16 pseudovirions generates therapeutic antitumor effects

We have previously demonstrated that C57BL/6 mice vaccinated with HPV-16 pseudovirions carrying OVA DNA were capable of preventing tumor growth upon challenge with OVA-expressing tumor [1]. To determine if DNA delivered by HPV-16 pseudovirions could generate appreciable therapeutic antitumor effects, we performed in vivo tumor treatment experiments. C57BL/6 mice were inoculated subcutaneously with B16/OVA tumor cells and treated with the various vaccination groups three days later. Mice were boosted with the same regimen on day 10 and 17 after tumor inoculation. As shown in Figure 3, tumor-bearing mice treated with HPV-16/OVA psV demonstrated significantly reduced tumor volume as compared to tumor-bearing mice treated with HPV-16 pseudovirions carrying DNA encoding irrelevant protein (GFP) or untreated mice. Thus, our data suggest that treatment with HPV-16 pseudovirions carrying OVA DNA can generate therapeutic antitumor effects against OVA-expressing tumors in tumor-bearing mice.

Vaccination with DNA delivered by HPV-16 pseudovirions generates the highest levels of antigen-specific CD8+ T cell immune responses compared to vaccination with DNA delivered by other methods

We next compared the OVA-specific CD8+ T cell immune responses generated by vaccination with OVAspecific DNA vaccines delivered by different methods including intramuscular injection followed by electroporation, gene gun, and HPV-16 pseudovirions. As shown in Figure 4, C57BL/6 mice vaccinated subcutaneously with HPV-16/OVA psV generated the highest number of OVA MHC class I peptide (SIINFEKL)-specific CD8+ T cell immune responses among all vaccination groups. In addition, we observed that DNA vaccine delivered by intramuscular injection followed by electroporation and DNA vaccine delivered by gene gun both generated higher OVA-specific CD8+ T cell immune responses at a higher dose of DNA (2 ug) compared to a lower dose of DNA (50 ng). Furthermore, we observed that delivery of DNA vaccine by HPV-16 pseudovirions generated a significantly higher antigen-specific CD8+ T

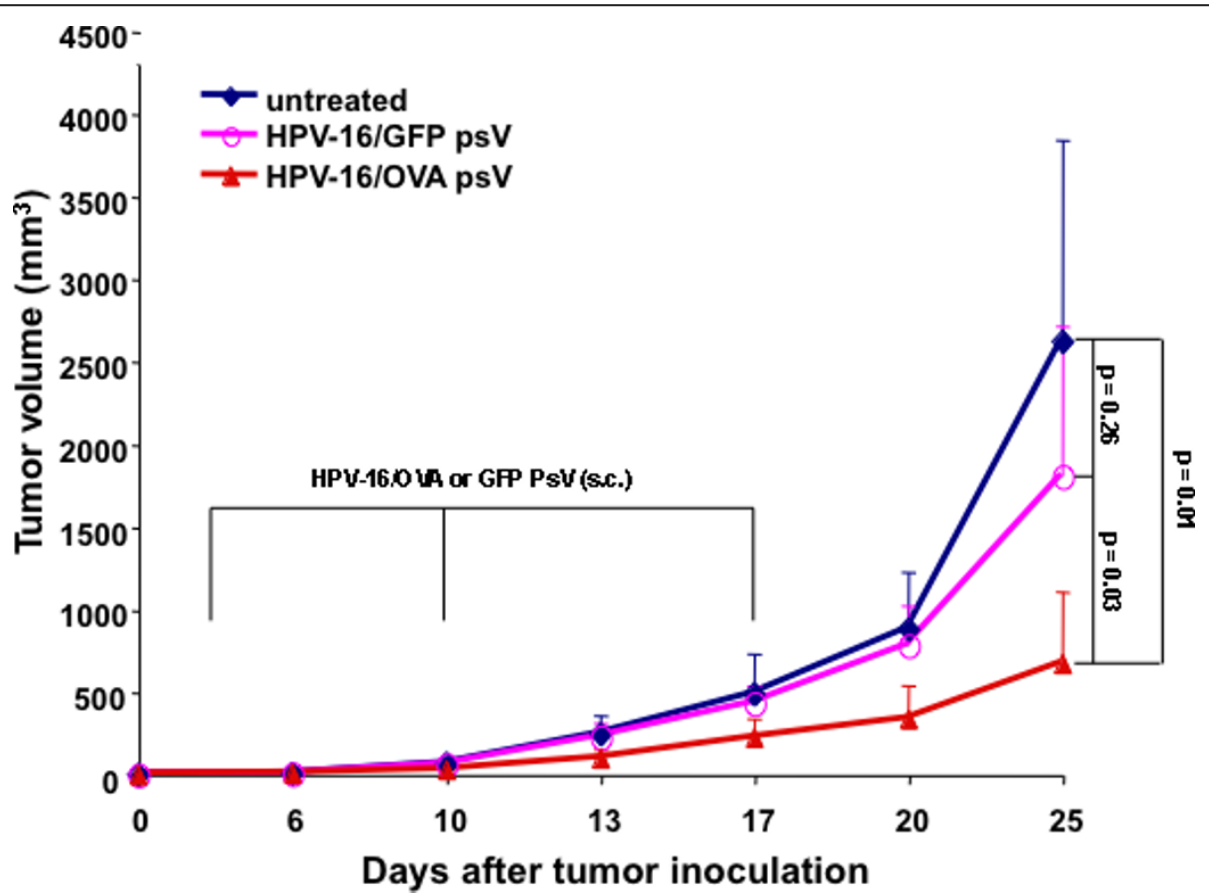

Figure 3 In vivo tumor treatment experiment with HPV-16 pseudovirion vaccination. 5-8 weeks old C57BL/6 mice (5 mice per group) were injected with $1 \times 10^{5}$ B16/OVA tumor cells subcutaneously. 3 days after tumor cell injection, the mice were vaccinated with either $5 \mu \mathrm{g}$ (total L1 protein amount) of HPV-16/OVA, or HPV-16/GFP pseudovirions via footpad injection. The mice were boosted with the same regimen on day 10 and 17. Tumor growth was monitored twice a week and tumor volume was calculated as described in the Material and Methods section. 


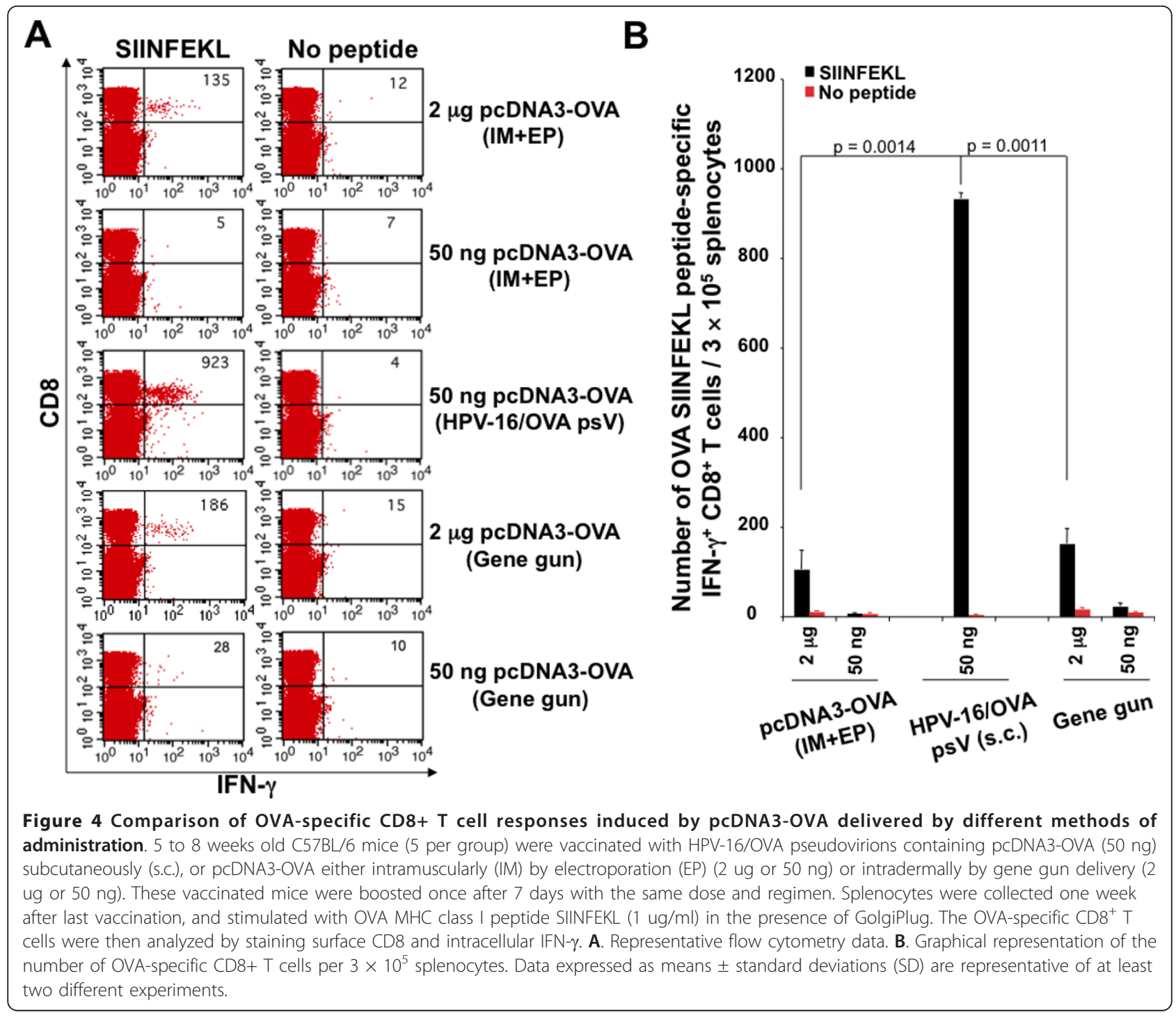

cell immune response even with a lower dose of DNA vaccine contained in the pseudovirion. These data suggest that antigen-specific vaccination with DNA vaccine delivered by pseudovirion represents one of the most promising approaches for generating antigen-specific $\mathrm{CD} 8+\mathrm{T}$ cell immune responses compared to other methods of delivering DNA in vivo.

HPV-16 pseudovirions generate the highest level of antigen-specific CD8+ T cell immune responses compared to other forms of antigen-specific vaccines

In addition to the many vaccine delivery methods that are currently being explored, there exist many forms of vaccination for antigen-specific immunotherapy. It is important to evaluate the efficacy of DNA vaccines delivered by HPV pseudovirions compared to other forms of antigen-specific vaccines for their ability to generate antigen-specific CD8+ T cells. We therefore compared the antigen-specific CD8 $+\mathrm{T}$ cell immune responses generated by HPV-16/OVA psV with other forms of vaccination including peptide-based (OVA8 (aa257-264) in incomplete Freund's adjuvant (IFA) and OVA30 (aa241-270) in IFA), protein-based (OVA protein in IFA), dendritic cell-based (OVA8-pulsed BMDCs) and vaccinia viral vector-based vaccination expressing OVA (OVA-VV). As shown in Figure 5, mice vaccinated subcutaneously with HPV-16/OVA psV generated the highest level of OVA-specific CD8+ T cell immune responses among all forms of OVA-specific vaccines. These data support that antigen-specific vaccination with DNA vaccine delivered by pseudovirion generates the best antigen-specific CD8+ T cell immune responses compared to other forms of antigen-specific vaccines. 


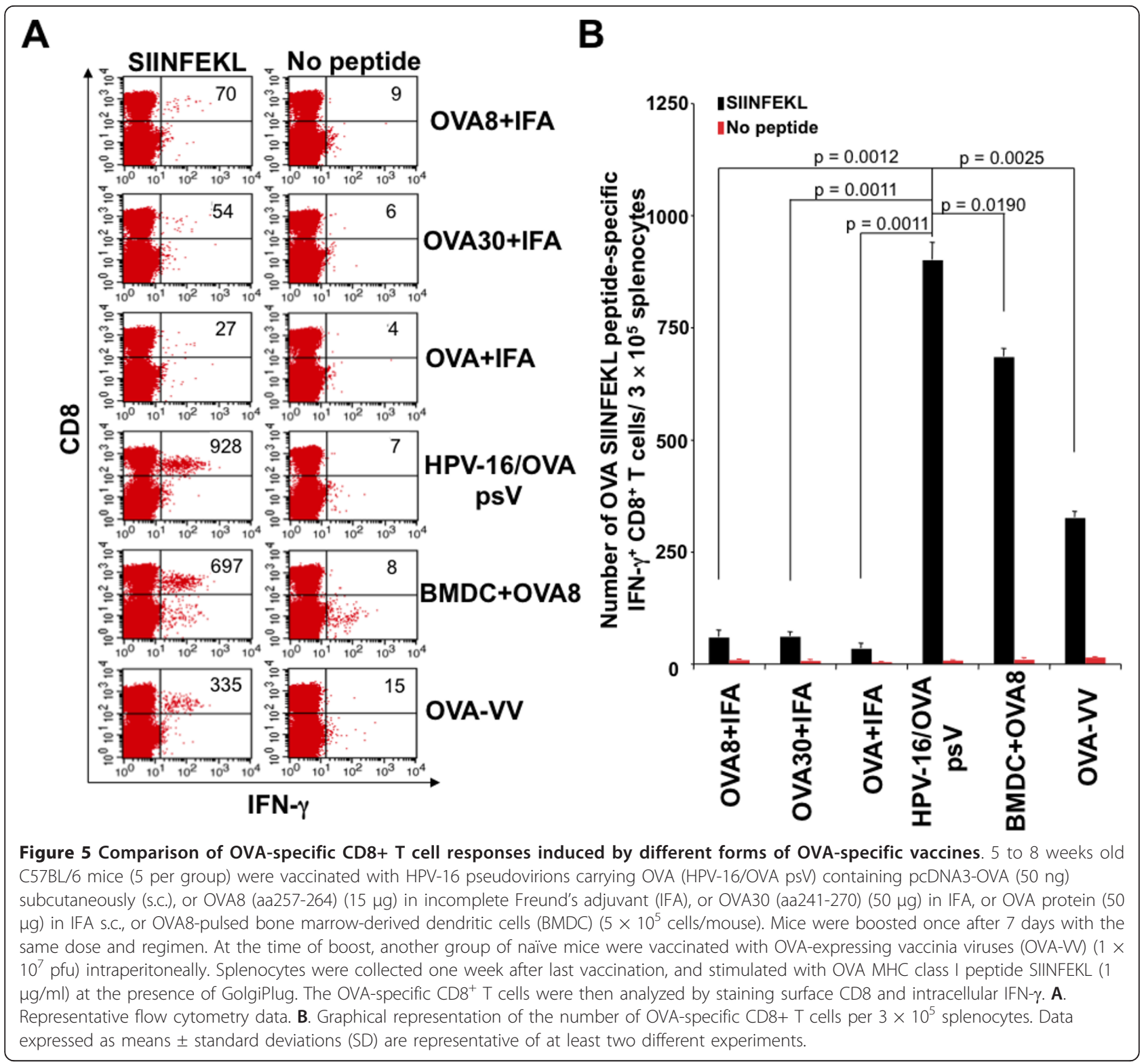

HPV pseudovirions is capable of delivering DNA encoding clinically relevant antigen to generate enhanced antigenspecific CD8+ T cell immune responses

In order to explore whether DNA delivered by HPV pseudovirions can be applied to clinically relevant antigens, we have chosen telomerase reverse transcriptase (TERT) (for review, see [8]). TERT is an endogenous antigen that is usually not expressed in most normal human somatic tissues but is reactivated in $85 \%$ of tumors. Upon reactivation of telomerase in tumor cells, TERT is processed and presented on the MHC class I molecules of tumor cells. Hence, TERT represents an attractive target for vaccine development. We therefore created DNA encoding calreticulin (CRT) linked to TERT198 minigene (aa198-205, VGRNFTNL)
[9] delivered by HPV pseudovirions. CRT has been shown to be one of the most potent intracellular targeting strategies to enhance antigen-specific CD8+ T cell immune responses in our previous study [10]. TERT198 minigene was shown to have a significantly high avidity for binding with $\mathrm{H}-2 \mathrm{~K}^{\mathrm{b}}$ [9]. As shown in Figure 6, mice vaccinated with HPV-16 pseudovirions carrying CRT linked to TERT198 minigene (HPV-16CRT/TERT198) demonstrated significantly higher number of TERT198-specific CD8+ T cell immune responses compared to mice vaccinated with control HPV-16 pseudovirions carrying CRT DNA. Thus, the delivery system of HPV-16 pseudovirions can be applied to other DNA vaccines targeting clinically relevant antigenic systems. 


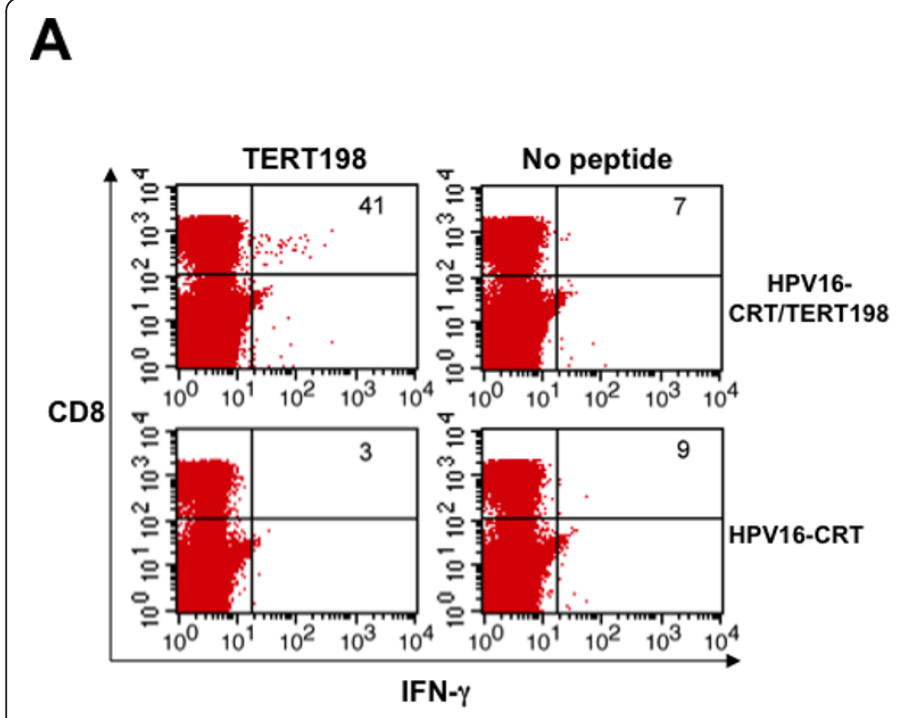

\section{B}

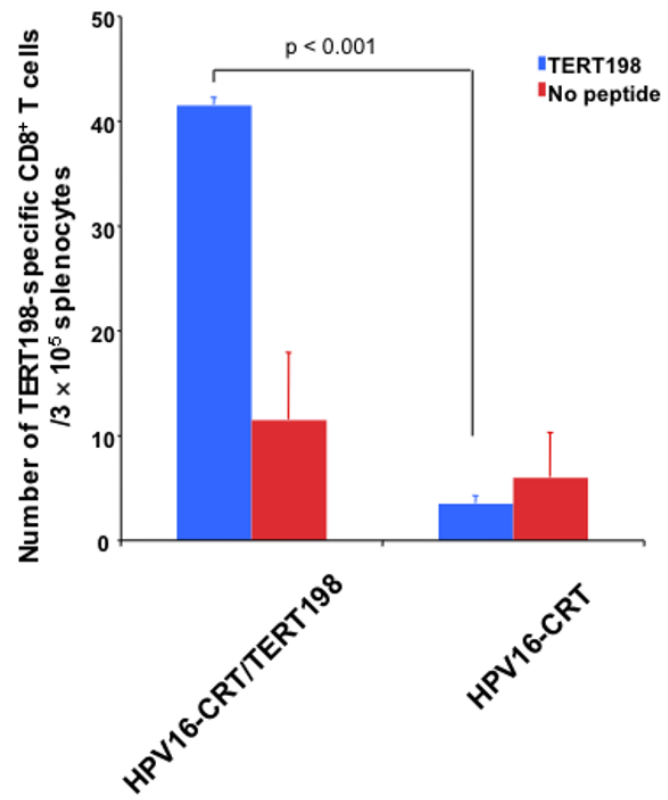

Figure 6 Intracellular cytokine staining followed by flow cytometry analysis for TERT198-specific CD8+ T cell immune responses. 5-8 weeks old C57BL/6 mice were vaccinated with $5 \mu \mathrm{g}$ of L1 protein of HPV16-CRT/TERT198, or HPV16-CRT pseudovirions via footpad injection, and boosted twice at 4-day interval with the same regimen. 1 week after last vaccination, splenocytes were prepared and stimulated with TERT198 peptide $(1 \mu \mathrm{g} / \mathrm{ml})$ at the presence of GolgiPlug overnight at 37 C. The TERT198-specific CD8+ T cells were then analyzed by staining surface CD8 and intracellular IFN- $\gamma$. A. Representative flow cytometry data. B. Graphical representation of the number of TERT198-specific CD8+ T cells per 3 $\times 10^{5}$ splenocytes. Data expressed as means \pm standard deviations (SD) are representative of at least two different experiments.

\section{Discussion}

In the current study, we found that replication-defective HPV pseudovirions prepared by intracellular assembly can infect cells with greater efficiency than HPV pseudovirions prepared by in vitro assembly. Our data also indicate that HPV-16 pseudovirions are capable of infecting DC-1 dendritic cell line in vitro. We also demonstrate that tumor-bearing mice treated with HPV-16/OVA psV could generate therapeutic antitumor effects against OVA-expressing tumors. In addition, we demonstrate that HPV pseudovirions carrying DNA encoding antigen generate the highest levels of antigenspecific CD8+ T cell immune responses compared to DNA delivered by other methods, including gene gun and intramuscular injection followed by electroporation. We also show that HPV pseudovirions generate the highest level of antigen-specific CD8+ $\mathrm{T}$ cell immune responses compared to other forms of antigen-specific vaccines. Furthermore, HPV pseudovirions could be used to deliver clinically relevant antigen for the generation of antigen-specific CD8+ T cell immune responses.

We observed that there is significantly higher infectivity in HPV pseudovirions generated by intracellular assembly compared to HPV pseudovirions prepared by in vitro assembly (Figure 1). The method of HPV pseudovirion generated by intracellular assembly resembles a system more biologically similar to the natural environment for viral assembly. Therefore, HPV pseudovirions generated by intracellular assembly will likely be more efficient at packaging the naked DNA into the L1 and L2 capsid proteins versus that of HPV pseudovirions generated by in vitro assembly. Furthermore, the conformation of L1 and L2 capsid proteins in the HPV pseudovirions generated by intracellular assembly will likely be better preserved compared to HPV pseudovirions generated by in vitro assembly. The in vitro assembly requires the disruption of L1 and L2 capsid proteins and refolding, a process during which may result in a conformational change of L1 and L2 capsid proteins. It has been shown that L2 is essential for the infectivity of the HPV pseudovirion $[1,11,12]$. These factors may account for the poorer infectivity of HPV pseudovirions generated by in vitro assembly compared to HPV pseudovirions generated by intracellular assembly.

Intradermal administration by gene gun and intramuscular injection followed by electroporation represent two state-of-the-art methods for DNA vaccine delivery. There have also been several clinical trials using gene 
gun or electroporation for therapeutic HPV DNA vaccines [13-15]. It is therefore important for future clinical translation to perform a head-to-head comparison to determine whether vaccination with HPV pseudovirion carrying DNA will be better than the aforementioned DNA vaccine delivery techniques at generating robust antigen-specific CD8+ T cell immune responses. We have previously compared the antigen-specific immune responses generated by HPV-16-pseudovirion carrying DNA encoding model antigen OVA with naked DNA vaccine encoding the same model antigen administered by gene gun [1]. Mice vaccinated with HPV-16/OVA pseudovirions generated significantly higher number of OVA-specific CD8+ T cell immune responses compared to mice vaccinated with naked OVA DNA via gene gun. In the current study, we found that vaccination with HPV-16/OVA pseudovirions generated the highest levels of antigen-specific CD8+ T cell immune responses compared to intradermal administration via gene gun and intramuscular injection followed by electroporation (Figure 4). Furthermore, delivery of DNA vaccine by HPV16/OVA pseudovirions generated a significantly greater OVA-specific CD8+ T cell immune response even with a lower dose of OVA DNA contained in the pseudovirion. Thus, DNA vaccine delivered by pseudovirion represents a more potent method to deliver naked DNA vaccine in vivo compared to gene gun and electroporation for their ability to generate antigen-specific immune responses.

We also compared DNA vaccine delivered by pseudovirions to other forms of antigen-specific vaccines including peptide-based vaccination, protein-based vaccination, dendritic cell-based vaccination and viral vector-based vaccination. We found that HPV-16/OVA pseudovirions generated the highest level of OVA-specific CD8+ T cell immune responses compared to other established forms of vaccination in the conditions tested (Figure 5). However, one of the major limitations for such kind of approach is that the forms of vaccines compared may not be optimized, including the conditions used for HPV pseudovirions. Therefore, the optimization of vaccine potency for each form of the vaccine before we perform a head-to-head comparison in the future will generate a more comprehensive picture of the efficacy of the different vaccination approaches compared to HPV pseudovirions.

There are several points of consideration for the clinical translation of HPV pseudovirions (for review, see [16]). For example, it is important to consider the type of HPV pseudovirion used to deliver the DNA vaccine. Currently, the commercially available prophylactic HPV vaccines use virus-like particles (VLPs) that include HPV types 16, 18, and/or HPV types 6 and 11. Vaccinations with HPV VLPs have been shown to generate potent type-specific neutralizing antibodies, which can inhibit subsequent infection of the same type of human papillomavirus. Thus, to avoid inhibition of vaccine efficacy by pre-existing immunity with this preventive HPV vaccine, it is essential to consider a different type of papillomavirus for pseudovirion delivery of DNA vaccine. This has broad clinical implications for delivering therapeutic HPV DNA vaccines.

\section{Conclusions}

In summary, HPV pseudovirions carrying DNA vaccine represent a significantly more efficient system compared to other methods of DNA vaccine delivery and other forms of vaccination for generating antigen-specific immunity. DNA vaccines delivered by HPV pseudovirions combine both the safety features of naked DNA and the potent infectivity of viral vector vaccines without the disadvantages associated with each of them. Thus, gene delivery using HPV pseudovirion technology represents a potentially promising non-viral gene delivery system to trigger potent immune responses against viral infections and cancer.

\section{Methods}

\section{Mice}

C57BL/6 mice (5-to-8-weeks old) were purchased from National Cancer Institute (Frederick, MD, USA). All animals were maintained under specific pathogen-free conditions, and all procedures were performed according to approved protocols and in accordance with recommendations for the proper use and care of laboratory animals.

\section{Cells}

293TT cells were generated by transfecting 293T cells with an additional copy of the SV40 large T antigen and were kindly provided by J. Schiller (NCI, NIH) [6]. 293TT cells were grown in complete Dulbecco's modified Eagle medium (DMEM) (Invitrogen) containing $10 \%$ heat-inactivated fetal bovine serum (Gemini BioProducts). DC-1 dendritic cell line has been described previously [17].

\section{Peptides, antibodies and reagents}

The H-2K ${ }^{\mathrm{b}}$-restricted Ovalbumin (OVA) peptide, SIINFEKL, was synthesized by Macromolecular Resources (Denver, CO) at a purity of $\geq 80 \%$. FITC-conjugated rat anti-mouse IFN- $\gamma$, PE-conjugated anti-mouse CD8, PECy5 conjugated anti-mouse B220, and APC-conjugated anti-mouse $\mathrm{CD} 11 \mathrm{c}$ antibodies were purchased from $\mathrm{BD}$ Pharmingen (BD Pharmingen, San Diego, CA). A horseradish peroxidase-conjugated rabbit anti-mouse immunoglobulin G (IgG) antibody was purchased from Zymed (San Francisco, CA). Peptides were generated as 
described before [18]. The following dominant minimal CTL peptide was used: OVA aa257-264 (OVA8). In addition, the long peptide deduced from the natural sequence of OVA protein was used: CTL peptide OVA aa241-270 SMLVLLPDEVSGLEQLESIINFEKLTEWTS (OVA30). OVA protein was purchased from Sigma. Incomplete Freund's adjuvant was purchased from Difco Laboratories. The vaccinia virus expressing the fulllength chicken ovalbumin (OVA) was generated using methods described previously $[19,20]$.

\section{Plasmid construction}

The plasmids encoding HPV16 and L1 and L2 (pShell16, p16L1 and p16L2) were kindly provided by Dr. John Schiller (NCI). The generation of ovalbuminexpressing plasmid (pcDNA3-OVA) and GFP-expressing plasmid (pcDNA3-GFP) has been described previously $[21,22]$. The generation of pcDNA3-CRT has been described previously [23]. For the generation of pcDNA3-CRT/TERT198, the synthesized oligos (AATTCgtgggcaggaatttcactaacctttgaA and AGCTTtcaaaggttagtgaaattcctgccacG) were annealed and subsequently cloned into EcoRI and HindIII sites of pcDNA3-CRT. The accuracy of the constructs were confirmed by DNA sequencing.

\section{HPV pseudovirion production}

For the generation of HPV pseudovirions using in vitro assembly, 293TT cells were transfected with pShell plasmid expressing codon-optimized HPV-16 L1, L2 capsid proteins only (without pcDNA3-GFP) using previously described protocols. The HPV structural capsid proteins have the ability to self-assemble into virus-like particles (VLPs). In vitro assembly into HPV pseudovirions involves the disruption and refolding of HPV-16 L1L2 VLPs $[4,5]$. Briefly, $5 \mu \mathrm{g}$ of purified HPV-16 L1L2 VLPs were incubated in $50 \mathrm{mM}$ Tris-HCL buffer ( $\mathrm{pH} 7.5$ ) containing $150 \mathrm{mM} \mathrm{NaCl}, 10 \mathrm{mM}$ EGTA and $20 \mathrm{mM}$ dithiothreitol (DTT) in a final volume of $100 \mu \mathrm{l}$ at room temperature (RT) for 30 minutes. $1 \mu \mathrm{g}$ of pcDNA3-GFP plasmid in $50 \mathrm{mM}$ Tris-HCL buffer and $150 \mathrm{mM} \mathrm{NaCl}$ was added to the disrupted VLPs at this step. The preparations were diluted with $25 \mathrm{mM} \mathrm{CaCl}_{2}$ and $20 \%$ dimethyl sulfoxide in equal volume at RT for 1 hour, and then treated with $10 \mathrm{U}$ of Benzonase Nuclease for 1 hour at RT to remove un-encapsidated plasmids.

For the generation of HPV pseudovirions by intracellular assembly, HPV-16 pseudovirions were made as described previously [6]. Briefly, 293TT cells were cotransfected with pShell plasmid expressing codon-optimized HPV-16 L1, L2 proteins and pcDNA3-GFP using Lipofectamine 2000 (Invitrogen, Carlsbad, CA). After 44 hours incubation, the cells were harvested and washed with Dulbecco's PBS (Invitrogen) supplemented with 9.5
$\mathrm{mM} \mathrm{MgCl} 2$ and antibiotic-antimycotic mixture (DPBS$\mathrm{Mg}$ ) (Invitrogen). The cells were suspended in DPBS$\mathrm{Mg}$ supplemented with 0.5\% Briji58, 0.2\% Benzonase (Novagen), 0.2\% Plasmid Safe (Epicentre) at > $100 \times 10^{6}$ cells $/ \mathrm{ml}$ and incubated at $37^{\circ} \mathrm{C}$ for 24 hours for capsid maturation. After maturation, the cell lysate was chilled on ice for 10 minutes. The salt concentration of the cell lysate was adjusted to $850 \mathrm{mM}$ and incubated on ice for 10 minutes. The lysate was then clarified by centrifugation, and the supernatant was then layered onto an Optiprep gradient. The gradient was spun for 4.5 hours at $16^{\circ} \mathrm{C}$ at $40,000 \mathrm{rpm}$ in a SW40 rotor (Beckman). The purity of HPV pseudovirions was evaluated by running the fractions on 4-15\% gradient SDS-PAGE gel. The encapsulated DNA plasmid was quantified by extracting encapsidated DNA from Optiprep factions followed by quantitative real time PCR compared to serial dilutions of naked DNA as described in [1]. The concentration of pcDNA3 plasmid DNA and pcDNA3-OVA DNA in the pseudovirions was determined to be approx. $6.2 \mathrm{ng}$ of DNA per $1 \mu \mathrm{g}$ of L1 protein.

\section{Generation of bone marrow-derived dendritic cells}

Bone marrow-derived dendritic cells (BMDCs) were generated from bone marrow progenitor cells as described previously [24]. Briefly, bone marrow cells were flushed from the femurs and tibiae of 5 - to 8 week-old C57BL/6 mice. Cells were washed twice with RPMI-1640 after lysis of red blood cells and resuspended at a density of $1 \times 10^{6} / \mathrm{ml}$ in RPMI- 1640 medium supplemented with $2 \mathrm{~m} M$ glutamine, $1 \mathrm{~m} M$ sodium pyruvate, $100 \mathrm{~m} M$ nonessential amino acids, $55 \mu M \beta$ mercaptoethanol, $100 \mathrm{IU} / \mathrm{ml}$ penicillin, $100 \mathrm{~g} / \mathrm{ml}$ streptomycin, $5 \%$ fetal bovine serum, and $20 \mathrm{ng} / \mathrm{ml} \mathrm{recombi-}$ nant murine GM-CSF (PeproTech, Rock Hill, NJ). The cells were then cultured in a 24-well plate $(1 \mathrm{ml} /$ well $)$ at $37^{\circ} \mathrm{C}$ in $5 \%$ humidified $\mathrm{CO}_{2}$. The wells were replenished with fresh medium supplemented with $20 \mathrm{ng} / \mathrm{ml}$ recombinant murine GM-CSF on days 2 and 4. The cells were harvested as indicated.

\section{In vitro infection with HPV pseudovirions}

DC- 1 cells $\left(5 \times 10^{4}\right.$ cells/well $)$ were seeded into 24 -well plate the night before infection. The seeded DC-1 cells were then infected with HPV16-GFP pseudovirions (L1 protein amount ranging from $0 \mathrm{ug}$ to $5 \mathrm{ug}$ ). 72 hours later, the cells were analyzed for GFP expression by flow cytometry.

\section{Vaccination with HPV pseudovirions}

C57BL/6 mice (5 per group) were vaccinated with indicated HPV pseudovirions (adjusted to $5 \mu \mathrm{g} \mathrm{L1}$ protein amount) subcutaneous injection at both hind footpads. 7 days later, the mice were boosted with indicated HPV 
pseudovirions with the same dose and regimen. For antigen-specific $\mathrm{T}$ cell detection, mouse splenocytes were harvested 1 week after last vaccination.

\section{Intradermal DNA vaccination via gene gun}

Gene gun particle-mediated DNA vaccination was performed as described previously [25]. Gold particles coated with pcDNA3-OVA or pcDNA3 were delivered to the shaved abdominal regions of mice by using a helium-driven gene gun (Bio-Rad Laboratories Inc., Hercules, Calif.) with a discharge pressure of $400 \mathrm{lb} / \mathrm{in}^{2}$. C57BL/ 6 mice (5 per group) were immunized with $2 \mu \mathrm{g}$ of the DNA vaccine and boosted with the same dose and regimen 1 week later. Splenocytes were harvested 1 week after the last vaccination.

\section{Intramuscular DNA vaccination with electroporation}

Electroporation-mediated DNA vaccination was performed with methods similar to those described by Jacob et al. [26]. C57BL/6 mice (5 per group) were injected in the tibialis muscle of the shaved hind leg. The appropriate concentration of DNA plasmid was diluted in a total volume of $20 \mu \mathrm{L}$ of PBS. DNA injection was followed immediately by square wave electroporation at the injection site using a BTX830 (BTX Harvard Apparatus, Holliston, MA). A tweezers electrode was used to deliver eight pulses at $100 \mathrm{~V}$ for 20 ms. Vaccinated mice were boosted with the same dose and regimen on the contralateral leg 1 week later. Splenocytes were harvested 1 week after the last vaccination.

\section{Comparison Vaccinations}

Peptide vaccination was performed using subcutaneous injection of $15 \mu \mathrm{g}$ of OVA8 (aa257-264) in incomplete Freund's adjuvant (IFA, Difco Laboratories) $(50 \% \mathrm{v} / \mathrm{v})$, or $50 \mu \mathrm{g}$ of OVA30 (aa241-270) in IFA in a total volume of $200 \mu \mathrm{L}$ [27]. Protein-based vaccination was performed using subcutaneous injection of $50 \mu \mathrm{g}$ of OVA protein in IFA similar to methods described previously [28]. Dendritic cell-based vaccination was performed using subcutaneous injection with $5 \times 10^{5}$ OVA8-pulsed bone marrow-derived dendritic cells (BMDC) similar to methods described previously [24]. Vaccinia-based vaccination was performed as described previously [29] using intraperitoneal injection with $1 \times 10^{7}$ pfu of OVAexpressing vaccinia viruses (OVA-VV) in $200 \mu \mathrm{L}$ PBS. C57BL/6 mice (5 per group) were vaccinated with OVA8 in IFA, OVA30 in IFA, OVA protein in IFA, or OVA8-pulsed BMDCs. Vaccinated mice were boosted 1 week later at the same dose and regimen. At the same time of boost, a separate set of C57BL/6 mice were intraperitoneally injected with OVA-VV. Splenocytes were harvested 1 week after last vaccination.
Intracellular cytokine staining and flow cytometry analysis Pooled splenocytes from each vaccination group were incubated for 20 hours with $1 \mu \mathrm{g} / \mathrm{ml}$ of OVA SIINFEKL peptide or TERT198 peptide (aa198-205) (VGRNFTNL) [9] in the presence of GolgiPlug (BD Pharmingen, San Diego, CA). The stimulated splenocytes were washed once with FACScan buffer, stained with PE-conjugated monoclonal rat antimouse CD8a (clone 53.6.7), and subjected to intracellular cytokine staining using the Cytofix/Cytoperm kit according to the manufacturer's instructions (BD Pharmingen, San Diego, CA). Intracellular IFN- $\gamma$ was stained with FITC-conjugated rat antimouse IFN- $\gamma$ (clone XMG1.2). Flow cytometry analysis was performed using FACSCalibur with CELLQuest software (BD Biosciences, Mountain View, CA).

\section{In vivo tumor experiments}

The OVA-expressing B16/OVA murine tumor model has been described previously [30]. 5-8 weeks old C57BL/6 mice (five mice per group) were injected with $1 \times 10^{5}$ B16/OVA tumor cells subcutaneously. 3 days after tumor cell inoculation, the tumor-bearing mice were treated with either $5 \mu \mathrm{g}$ (total L1 protein amount) of HPV16-OVA, or HPV16-GFP pseudovirions via footpad injection. Tumor-bearing mice were boosted with the same regimen on day 10 and 17 after tumor challenge. Tumor-bearing mice without treatment were included as a control. Tumor growth was monitored twice a week. Tumor volumes were evaluated using the formula $\mathrm{V}\left(\mathrm{mm}^{3}\right)=3.14$ [largest diameter $\times$ (perpendicular diameter $\left.)^{2}\right] / 6$.

\section{Statistical analysis}

Data expressed as means \pm standard deviations (SD) are representative of at least two different experiments. Comparisons between individual data points were made by 2 -tailed Student's $t$ test. A $p$ value $<0.05$ was considered significant.

\section{List of Abbreviations}

(BMDCs): Bone marrow-derived dendritic cells; (HPV): Human papillomavirus; (OVA): Ovalbumin; (psV): pseudovirions; (VLPs): virus-like particles.

\section{Acknowledgements}

We would like to thank Richard Roden for helpful discussions. We would also like to thank Janson Trieu and Ellen Axenfeld for critical review of this manuscript. This work was supported by 1 RO1 CA114425 01 (Wu) and SPORE programs (P50 CA098252 and P50 CA96784-06) of the National Cancer Institute.

\section{Author details}

'Department of Pathology, Johns Hopkins Medical Institutions, Baltimore, MD, USA. ²Department of Obstetrics and Gynecology, Johns Hopkins Medical Institutions, Baltimore, MD, USA. ${ }^{3}$ Department of Oncology, Johns Hopkins Medical Institutions, Baltimore, MD, USA. ${ }^{4}$ Department of Molecular Microbiology and Immunology, Johns Hopkins Medical Institutions, Baltimore, MD, USA. ${ }^{5}$ Department of Oncological Sciences, Mount Sinai School of Medicine, New York, NY, USA. 


\section{Authors' contributions}

SP was involved in the design of the study and acquired and analyzed the data. SHC analyzed and interpreted the data. BM and TCW analyzed and interpreted the data and drafted the manuscript. CFH and TCW conceived and designed the study as well as provided general supervision of the research group. All authors gave final approval of the manuscript.

\section{Competing interests}

The authors declare that they have no competing interests.

Received: 10 June 2011 Accepted: 28 July 2011 Published: 28 July 2011

\section{References}

1. Peng S, Monie A, Kang TH, Hung CF, Roden R, Wu TC: Efficient delivery of DNA vaccines using human papillomavirus pseudovirions. Gene Ther 2010, 17(12):1453-64

2. Yang R, Murillo FM, Cui H, Blosser R, Uematsu S, Takeda K, Akira S, Viscidi RP, Roden RB: Papillomavirus-like particles stimulate murine bone marrow-derived dendritic cells to produce alpha interferon and Th1 immune responses via MyD88. J Virol 2004, 78:11152-11160.

3. Yan M, Peng J, Jabbar IA, Liu X, Filgueira L, Frazer IH, Thomas R: Activation of dendritic cells by human papillomavirus-like particles through TLR4 and NF-kappaB-mediated signalling, moderated by TGF-beta. Immunology and cell biology 2005, 83:83-91.

4. Touze A, Coursaget $P$ : In vitro gene transfer using human papillomaviruslike particles. Nucleic Acids Res 1998, 26:1317-1323.

5. Shi W, Liu J, Huang Y, Qiao L: Papillomavirus pseudovirus: a novel vaccine to induce mucosal and systemic cytotoxic T-lymphocyte responses. $J$ Virol 2001, 75:10139-10148.

6. Buck CB, Pastrana DV, Lowy DR, Schiller JT: Efficient intracellular assembly of papillomaviral vectors. J Virol 2004, 78:751-757.

7. Buck CB, Pastrana DV, Lowy DR, Schiller JT: Generation of HPV pseudovirions using transfection and their use in neutralization assays. Methods Mol Med 2005, 119:445-462.

8. Harley CB: Telomerase and cancer therapeutics. Nat Rev Cancer 2008, 8:167-179

9. Mennuni C, Ugel S, Mori F, Cipriani B, lezzi M, Pannellini T, Lazzaro D, Ciliberto G, La Monica N, Zanovello P, et al: Preventive vaccination with telomerase controls tumor growth in genetically engineered and carcinogen-induced mouse models of cancer. Cancer Res 2008, 68:9865-9874.

10. Kim JW, Hung CF, Juang J, He L, Kim TW, Armstrong DK, Pai SI, Chen PJ, Lin CT, Boyd DA, Wu TC: Comparison of HPV DNA vaccines employing intracellular targeting strategies. Gene Ther 2004, 11(12):1011-8.

11. Gambhira R, Jagu S, Karanam B, Day PM, Roden R: Role of L2 cysteines in papillomavirus infection and neutralization. Virol J 2009, 6:176.

12. Campos SK, Ozbun MA: Two highly conserved cysteine residues in HPV16 L2 form an intramolecular disulfide bond and are critical for infectivity in human keratinocytes. PLOS ONE 2009, 4:e4463.

13. Vaccine Therapy With or Without Imiquimod in Treating Patients With Grade 3 Cervical Intraepithelial Neoplasia. [http://clinicaltrials.gov/ct2/ show/NCT00788164].

14. Trimble C, Lin CT, Hung CF, Pai S, Juang J, He L, Gillison M, Pardoll D, Wu L, Wu TC: Comparison of the CD8+ T cell responses and antitumor effects generated by DNA vaccine administered through gene gun, biojector, and syringe. Vaccine 2003, 21:4036-4042.

15. Trimble CL, Peng S, Kos F, Gravitt P, Viscidi R, Sugar E, Pardoll D, Wu TC: A phase I trial of a human papillomavirus DNA vaccine for HPV16+ cervical intraepithelial neoplasia 2/3. Clin Cancer Res 2009, 15:361-367.

16. Ma B, Roden RBS, Hung CF, Wu TC: HPV pseudovirions as DNA delivery vehicles. Therapeutic Delivery 2011, 2:427-430.

17. Kim TW, Hung CF, Boyd DA, He L, Lin CT, Kaiserman D, Bird PI, Wu TC: Enhancement of DNA vaccine potency by coadministration of a tumor antigen gene and DNA encoding serine protease inhibitor-6. Cancer Res 2004, 64:400-405.

18. Feltkamp MC, Smits HL, Vierboom MP, Minnaar RP, de JB, Drijfhout JW, ter SJ, Melief CJ, Kast WM: Vaccination with cytotoxic T lymphocyte epitope-containing peptide protects against a tumor induced by human papillomavirus type 16-transformed cells. Eur I Immunol 1993, 23:2242-2249.
19. Norbury CC, Princiotta MF, Bacik I, Brutkiewicz RR, Wood P, Elliott T, Bennink JR, Yewdell JW: Multiple antigen-specific processing pathways for activating naive CD8+ T cells in vivo. J Immunol 2001, 166:4355-4362.

20. Zhang YQ, Tsai YC, Monie A, Wu TC, Hung CF: Enhancing the therapeutic effect against ovarian cancer through a combination of viral oncolysis and antigen-specific immunotherapy. Mol Ther 2010, 18:692-699.

21. Kim TW, Hung CF, Ling M, Juang J, He L, Hardwick JM, Kumar S, Wu TC: Enhancing DNA vaccine potency by coadministration of DNA encoding antiapoptotic proteins. J Clin Invest 2003, 112:109-117.

22. Hung C-F, Cheng W-F, Hsu K-F, Chai C-Y, He L, Ling M, Wu T-C: Cancer immunotherapy using a DNA vaccine encoding the translocation domain of a bacterial toxin linked to a tumor antigen. Cancer Research 2001, 61:3698-3703.

23. Cheng WF, Hung CF, Chai CY, Hsu KF, He L, Ling M, Wu TC: Tumor-specific immunity and antiangiogenesis generated by a DNA vaccine encoding calreticulin linked to a tumor antigen. J Clin Invest 2001, 108:669-678.

24. Peng S, Kim TW, Lee JH, Yang M, He L, Hung CF, Wu TC: Vaccination with dendritic cells transfected with BAK and BAX siRNA enhances antigenspecific immune responses by prolonging dendritic cell life. Hum Gene Ther 2005, 16:584-593.

25. Peng S, Hung C-F, Trimble C, He L, Yeatermeyer J, Boyd D, Wu T-C: Development of a DNA vaccine targeting HPV-16 oncogenic protein E6. J Virol 2004, 78:8468-8476.

26. Jacob J, Radkevich O, Forni G, Zielinski J, Shim D, Jones RF, Wei WZ: Activity of DNA vaccines encoding self or heterologous Her-2/neu in Her-2 or neu transgenic mice. Cell Immunol 2006, 240:96-106.

27. Bijker MS, van den Eeden SJ, Franken KL, Melief CJ, Offringa R, van der Burg SH: CD8+ CTL priming by exact peptide epitopes in incomplete Freund's adjuvant induces a vanishing CTL response, whereas long peptides induce sustained CTL reactivity. J Immunol 2007, 179:5033-5040.

28. Nelson D, Bundell C, Robinson B: In vivo cross-presentation of a soluble protein antigen: kinetics, distribution, and generation of effector CTL recognizing dominant and subdominant epitopes. J Immunol 2000, 165:6123-6132.

29. Chang CL, Ma B, Pang X, Wu TC, Hung CF: Treatment with cyclooxygenase-2 inhibitors enables repeated administration of vaccinia virus for control of ovarian cancer. Mol Ther 2009, 17:1365-1372.

30. Kang $T H$, Lee $J H$, Song $C K$, Han HD, Shin BC, Pai Sl, Hung CF, Trimble C, Lim JS, Kim TW, Wu TC: Epigallocatechin-3-gallate enhances CD8+ T cellmediated antitumor immunity induced by DNA vaccination. Cancer Res 2007, 67:802-811.

doi:10.1186/2045-3701-1-26

Cite this article as: Peng et al: DNA vaccines delivered by human papillomavirus pseudovirions as a promising approach for generating antigen-specific CD8+ T cell immunity. Cell \& Bioscience 2011 1:26.

\section{Submit your next manuscript to BioMed Central and take full advantage of:}

- Convenient online submission

- Thorough peer review

- No space constraints or color figure charges

- Immediate publication on acceptance

- Inclusion in PubMed, CAS, Scopus and Google Scholar

- Research which is freely available for redistribution

Submit your manuscript at www.biomedcentral.com/submit
Ciomed Central 